\title{
Enhancement of tensile strength and flexibility of Polycaprolactone/Tapioca starch blends by Octadecylamine modified clay.
}

\begin{abstract}
Polycaprolactone/tapioca starch/octadecylamine modified clay (OMMT) nanocomposites were successfully prepared by melt blending. X-ray diffraction and transmission electron microscopy (TEM) of the products showed that they are nanocomposites of a mixture intercalated and exfoliated types. In addition, the TEM also revealed that the OMMTlayers are homogeneously distributed in the polymer matrix. The presence of 1 php of OMMT improved the compatibility of the polymers in the blends which consequently increased the tensile strength of the blend of more than $60 \%$ and elongation at break of more than $1,000 \%$.
\end{abstract}

Keyword: Polycaprolactone; Tapioca starch; Modified clay. 